\title{
Article \\ Understanding the Reaction Crystallization Process of Glycidyl Trimethyl Ammonium Chloride
}

\author{
Shuai Yu ${ }^{1} \mathbb{D}$, Hui Chen ${ }^{1}$, Xujie Gao ${ }^{1}$, Weichun Feng ${ }^{2}$, Wenguo Xing ${ }^{2}$, Shichao Du ${ }^{1}$ (D, Yan Wang ${ }^{1}$, Fumin Xue ${ }^{1, *}$ \\ and Yan Cheng $1, *$
}

check for

updates

Citation: Yu, S.; Chen, H.; Gao, X.; Feng, W.; Xing, W.; Du, S.; Wang, Y.; Xue, F.; Cheng, Y. Understanding the Reaction Crystallization Process of Glycidyl Trimethyl Ammonium Chloride. Crystals 2021, 11, 449. https: / / doi.org/10.3390/ cryst11040449

Academic Editors: Sohrab Rohani, Zhenguo Gao, Junbo Gong, Huaiyu Yang and Brahim Benyahia

Received: 29 March 2021

Accepted: 16 April 2021

Published: 20 April 2021

Publisher's Note: MDPI stays neutra with regard to jurisdictional claims in published maps and institutional affiliations.

Copyright: (c) 2021 by the authors. Licensee MDPI, Basel, Switzerland. This article is an open access article distributed under the terms and conditions of the Creative Commons Attribution (CC BY) license (https:/ / creativecommons.org/licenses/by/ $4.0 /)$.
1 Shandong Analysis and Test Center, School of Pharmaceutical Sciences, Qilu University of Technology (Shandong Academy of Sciences), Jinan 250014, China; yushuai@qlu.edu.cn (S.Y.);

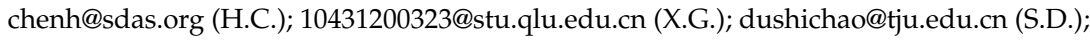
wangyan57@tju.edu.cn (Y.W.)

2 Chemical Technology Research Institute of Shandong, Qingdao University of Science \& Technology, Jinan 250014, China; lhgyfeng@vip.sina.com (W.F.); hg074@qust.edu.cn (W.X.)

* Correspondence: xuefumin@qlu.edu.cn (F.X.); chengy@sdatc.com.cn (Y.C.); Tel.: +86-0531-82605348 (F.X.); +86-13370579017 (Y.C.)

\begin{abstract}
Reaction crystallization to produce glycidyl trimethyl ammonium chloride (GTA) via epichlorohydrin with gas-state trimethylamine was investigated. The crystallization process of the GTA gas-liquid reaction was optimized by a seed method. The optimized technology can prepare GTA products with crystal form and purity greater than $97 \%$. The crystallization process of GTA consists of four steps (i.e., addition of seed, dispersion of seed, growth of crystals on the seed surfaces, agglomeration and growth of crystal). Seed method and flow rate are the key factors affecting purity. The purity and particle size of GTA crystals were satisfactory as long as the operation was kept within the defined envelope. The experiments were conducted on a $1 \mathrm{~L}$ reactor and successfully scaled-up to $3000 \mathrm{~L}$ in industry.
\end{abstract}

Keywords: glycidyl trimethyl ammonium chloride (GTA); reaction crystallization; purity; crystallization process optimization

\section{Introduction}

Glycidyl trimethyl ammonium chloride (GTA, CAS No. 3033-77-0), with a chemical formula $\mathrm{C}_{6} \mathrm{H}_{14} \mathrm{ClNO}$, is a kind of solid active cationic etherifying agent. GTA can react with a variety of substrates (i.e., starch, cellulose, guar gum, polyacrylamide and so on) to produce modified products [1]. Therefore, GTA is widely used in the industries of paper, daily chemicals, petroleum, and water treatment [2]. GTA is a white solid at room temperature and is very moisture absorbing. Thus, GTA generally needs to be stored in an airtight and cool place.

GTA is generally prepared by the reaction of epichlorohydrin with trimethylamine. The trimethylamine can be in the form of an aqueous solution, an alcohol solution, or hydrochloride. There is little research on the reaction crystallization process of gas trimethylamine as reactant. The reactive nature of the gas adds difficulty to the control of the reaction process [3-5]. Preparation of trimethylamine solution increases the production procedure and cost. Solubility and supersaturation are important data bases for crystallization process research [6-12]. However, it is difficult to determine the supersaturation of the reaction crystallization process, which brings challenges to the crystallization process control [13-15]. Previous literature mainly focused on the synthesis of GTA. There are relatively few studies on its crystallization process. The main problems in production of GTA are low purity, low crystallinity and difficulty in particle size control. Especially when epichlorohydrin is recycled, the product quality decreases obviously. Purity and particle size are important quality indexes of solid products [16]. The deep understanding of the crystallization process is also the basis of technological innovation and optimization [17-19]. 
In this study, epichlorohydrin and gas trimethylamine were considered as reactants. The crystallization process of GTA was studied based on the gas-liquid reaction. On the basis of further understanding of the GTA crystallization process, the application of a seed method of the GTA gas-liquid reaction crystallization process was proposed. The effects of operating parameters on the purity, recycling of reactants, particle size and other properties of the products were investigated on the basis of the seed method. An optimized GTA production process was obtained, which can be scaled up, and it contains industrial application value.

\section{Materials and Methods}

\subsection{Materials}

The details of the materials used in this experiment are listed in Table 1. All the materials were used without any further purification.

Table 1. Details of materials used in this experiment.

\begin{tabular}{|c|c|c|}
\hline Name & Supplier & Purity (wt \%) \\
\hline epichlorohydrin & $\begin{array}{l}\text { Sinopec Qilu petrochemical Company, Zibo } \\
\text { City, Shandong Province, China }\end{array}$ & $99 \%$ \\
\hline trimethylamine & $\begin{array}{l}\text { Sinopec Qilu petrochemical Company, Zibo } \\
\text { City, Shandong Province, China }\end{array}$ & $99 \%$ \\
\hline methyl tert-butyl ether & $\begin{array}{l}\text { Sinopharm Chemical Reagent Co., Ltd., } \\
\text { Shanghai City, China }\end{array}$ & $99 \%$ \\
\hline sodium hydroxide $(\mathrm{NaOH})$ & $\begin{array}{l}\text { Sinopharm Chemical Reagent Co., Ltd., } \\
\text { Shanghai City, China }\end{array}$ & $96 \%$ \\
\hline phenolphthalein & $\begin{array}{c}\text { Sinopharm Chemical Reagent Co., Ltd., } \\
\text { Shanghai City, China }\end{array}$ & indicator \\
\hline water & $\begin{array}{l}\text { arium }^{\circledR} \text { advance EDI, Sartorius, } \\
\text { Göttingen, Germany }\end{array}$ & ultrapure \\
\hline
\end{tabular}

\subsection{Equipment}

As shown in Figure 1, a $1 \mathrm{~L}$ glass jacketed reactor with mechanical agitation was selected (supplied by Pharmavision (Qingdao) Intelligent Technology Ltd., Qingdao City, Shandong Province, China). The temperature was controlled by a thermostatic watercirculating bath (CF41, Julabo, Seelbach, Germany) with an uncertainty of $0.05{ }^{\circ} \mathrm{C}$. A flowmeter was used to regulate the flow rate of gas. The sample of GTA solids was evaluated by X-ray powder diffraction (XRPD), which was carried out using an X'pert3 Powder (PANalytical B.V., Almelo, The Netherlands) instrument. $\mathrm{Cu} K \alpha$ radiation $(\lambda=1.5418 \mathrm{~nm})$ was used in the experiment, and the tube voltage and current were set at $40 \mathrm{kV}$ and $30 \mathrm{~mA}$, respectively. The data were measured from $5^{\circ}$ to $50^{\circ}(2 \theta)$ at a scan speed of $10^{\circ} / \mathrm{min}$ at room temperature $\left(25^{\circ} \mathrm{C}\right)$ under atmospheric pressure (about $101.3 \mathrm{kPa}$ ). Infrared spectroscopy (Bruker, Karlsruhe, Germany) was used to determine product. The thermal decomposition temperature of GTA was measured by thermogravimetric analysis (DSC-STA449 F3, NETZSH, Selb, Germany). A polarization microscope (OLYMPUS BX 53, Tokyo, Japan) was used to observe crystal morphology in this investigation. 


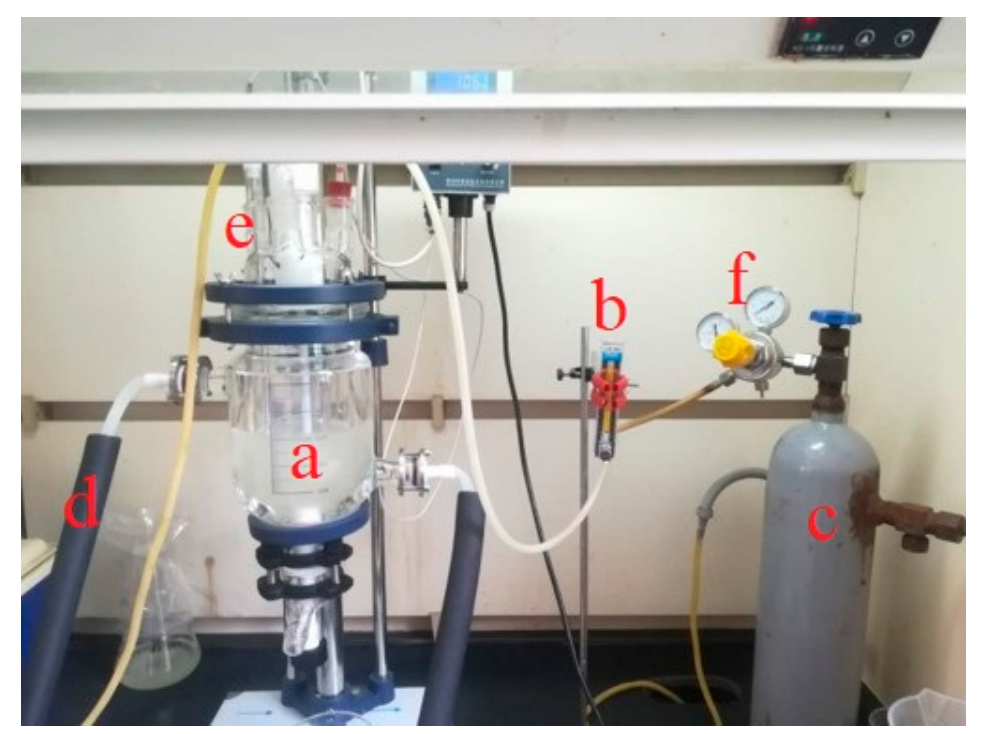

Figure 1. Diagram of crystallization system ( $a$ - reactor, $b$ - flowmeter, $c$ - gas cylinder, $\mathrm{d}$ - thermostatic water-circulating bath, e-thermometer, $\mathrm{f}$-relief valve).

\subsection{Crystallization Procedure}

The reactor was added with a certain amount of epichlorohydrin and kept at a fixed temperature for approximately half an hour. Mechanical agitation was initiated at a fixed rate of agitation during this time. The trimethylamine gas was injected into the system at a fixed speed for a certain period of time (e.g., $20 \mathrm{~min}$, ensure that there are no solids in the system). We stopped gas addition and continued stirring for $10 \mathrm{~min}$. Then, a certain quantity of seed was added to the system (e.g., $1 \mathrm{wt} \%$, with the theoretical mass of the product). Crystal seed was selected from the product with high purity and crystallinity (produced by spontaneous nucleation method), and was ground and crushed before use. After the seeds were suspended in the system for $20 \mathrm{~min}$, a certain amount of trimethylamine gas was added again at a fixed adding speed. Epichlorohydrin, used as a reactant and dispersant in the reaction process, was relatively excessive. After that, the solution was kept at a fixed temperature for 2-3 h with stirring. Then, the suspension was filtered, washed twice by methyl tert-butyl ether and dried in a vacuum oven at room temperature for $12 \mathrm{~h}$. The molar ratio of epichlorohydrin to trimethylamine is approximately $4: 1$ in the overall process.

\subsection{Content Determination}

A $1 \mathrm{~g}$ sample of GTA was accurately weighed with a balance (Model AL204, Mettler Toledo, Zurich, Switzerland; with a precision of $\pm 0.0001 \mathrm{~g}$ ) and placed in a $250 \mathrm{~mL}$ conical flask. Then, $20 \mathrm{~mL}$ solution of calcium chloride hydrochloride was added into the conical flask. The system was sealed and magnetically stirred for $20 \mathrm{~min}$. After that, $50 \mathrm{~mL}$ purified water and 3 drops of phenolphthalein were added to the system. The standard solution of $\mathrm{NaOH}(0.5 \mathrm{~mol} / \mathrm{L})$ was dropped until the solution turned pink, when the $\mathrm{pH}$ value is approximately 8.5 (measured by a pH meter, Sartorius PB-10, Göttingen, Germany). In addition, a blank titration sample is required. The difference of the blank sample is that there is no GTA added to the system. The purity value was calculated based on the following equation:

$$
\text { Purity }(\%)=\frac{\left(V_{\mathrm{NaOH}, \text { blank }}-V_{\mathrm{NaOH}, \text { sample }}\right) \times \mathrm{C}_{\mathrm{NaOH}} \times M_{\mathrm{GTA}}}{1000 \mathrm{~m}_{\text {sample }}} \times 100 \%
$$

where $V_{\mathrm{NaOH}}$, blank and $V_{\mathrm{NaOH}}$, sample $(\mathrm{mL})$ is the used volume of $\mathrm{NaOH}$ of the blank sample and the GTA sample, respectively; $\mathrm{C}_{\mathrm{NaOH}}(\mathrm{mol} / \mathrm{mL})$ denotes the concentration of $\mathrm{NaOH}$ 
solution; $m_{\text {sample }}(\mathrm{g})$ means the mass of GTA used in the measurement; $M_{G T A}(\mathrm{~g} / \mathrm{mol})$ means the relative molecular mass of GTA.

\section{Results and Discussion}

\subsection{Process Analysis of Crystallization}

The crystallization process of GTA is directly prepared by one-step reaction crystallization of trimethylamine in the gas phase and epichlorohydrin in the liquid phase. The reaction equation is shown in Figure 2. In general, different reaction conditions correspond to different degrees of side reactions. There is no seed-adding step before optimization. That is to say, GTA is prepared by a spontaneous nucleation method. Due to the fast spontaneous nucleation phenomenon, the crystallinity and particle size of solids production is uncontrollable. The product may be crystalline or amorphous solid or a mixture of them. The final product would not be crystalline if the initial product is amorphous. That is to say, the amorphous solid would not transform to crystal form during the reaction process in this experiment. The products obtained by the seed method were all crystals, and the crystal form product produced by the seed method (details are mentioned in the "Crystallization procedure" section; red line in Figure 3) is the same as the product of spontaneous nucleation (blue line in Figure 3). The main $2 \theta$ values were $16.595^{\circ}, 17.743^{\circ}$, $20.030^{\circ}, 20.997^{\circ}, 23.514^{\circ}, 25.189^{\circ}$ and $31.037^{\circ}$. According to the IR spectrum (Figure 4) and the TG curve (Figure 5). Although the solid state (crystal form or amorphous) of GTA products is different, there was no significant difference in the GTA substance itself. Regardless of crystal or amorphous product, decomposition temperature was $160^{\circ} \mathrm{C}$.

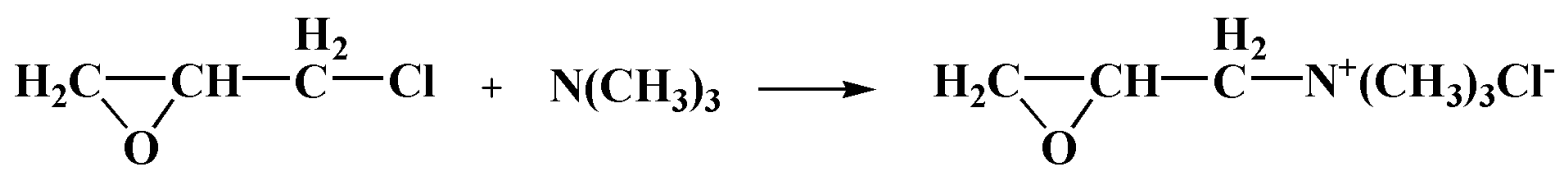

(GTA)

Figure 2. Reaction equation of GTA.

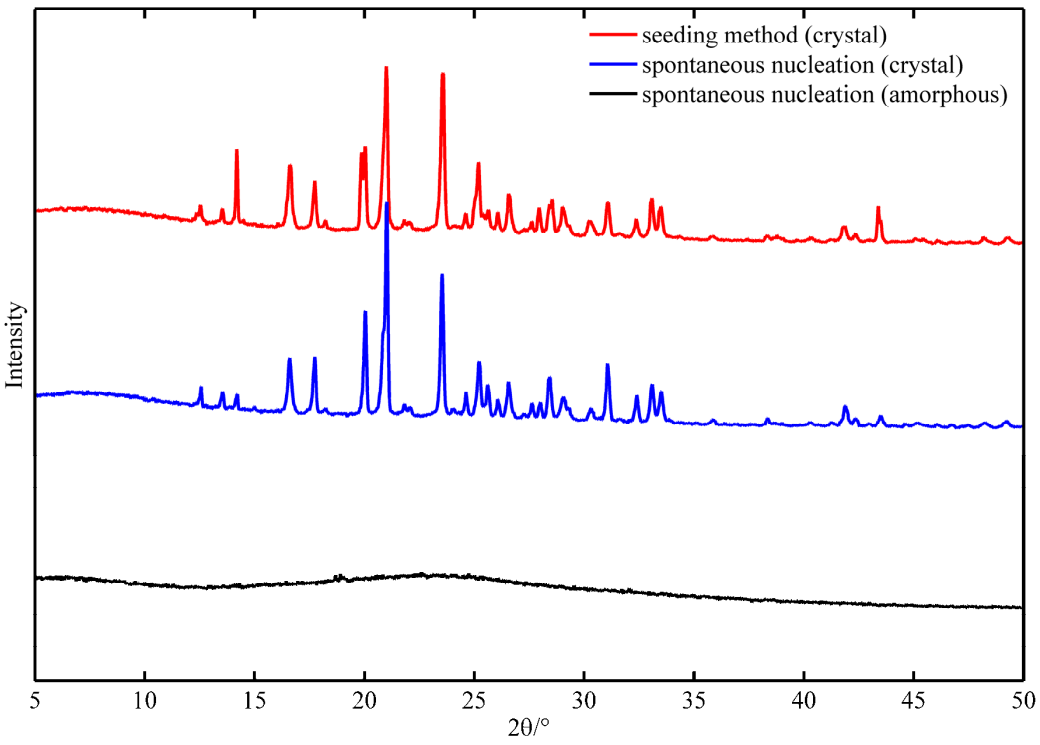

Figure 3. XRPD patterns of GTA product prepared by different methods. 


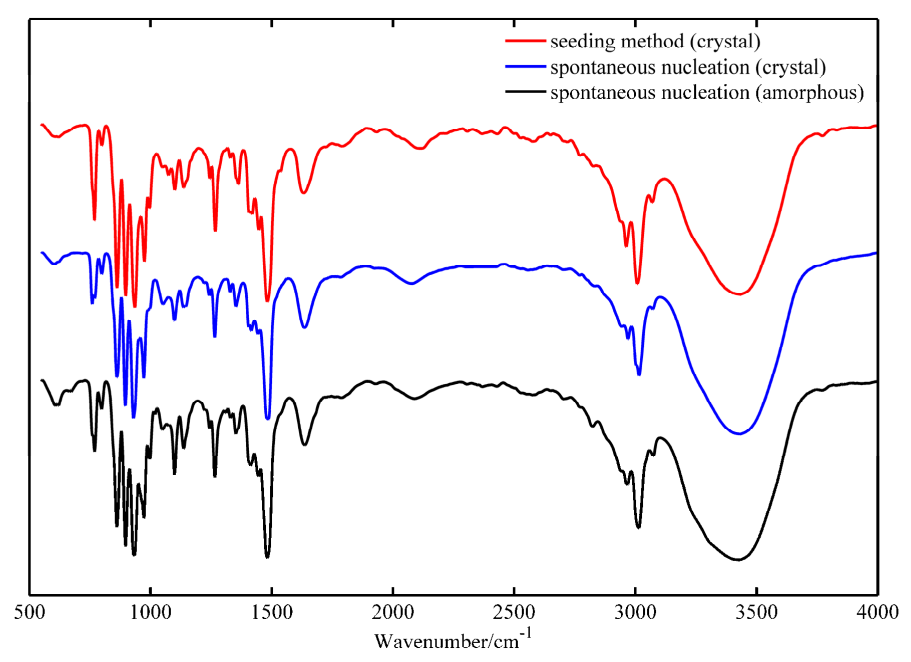

(a)

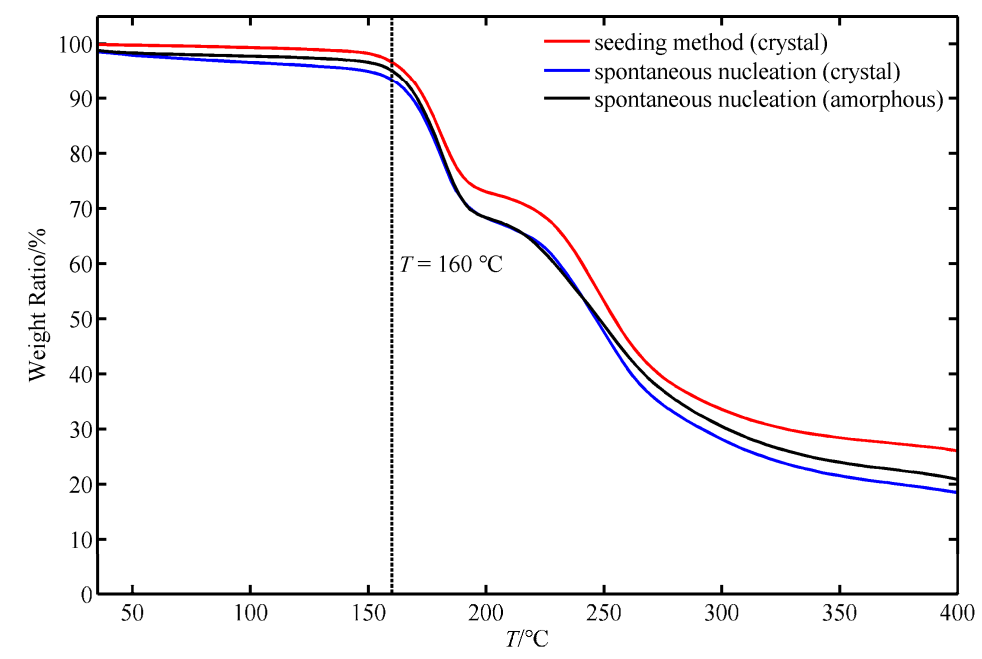

(b)

Figure 4. (a) IR spectrum of GTA product prepared by different methods. (b) TG curve of GTA product prepared by different methods.

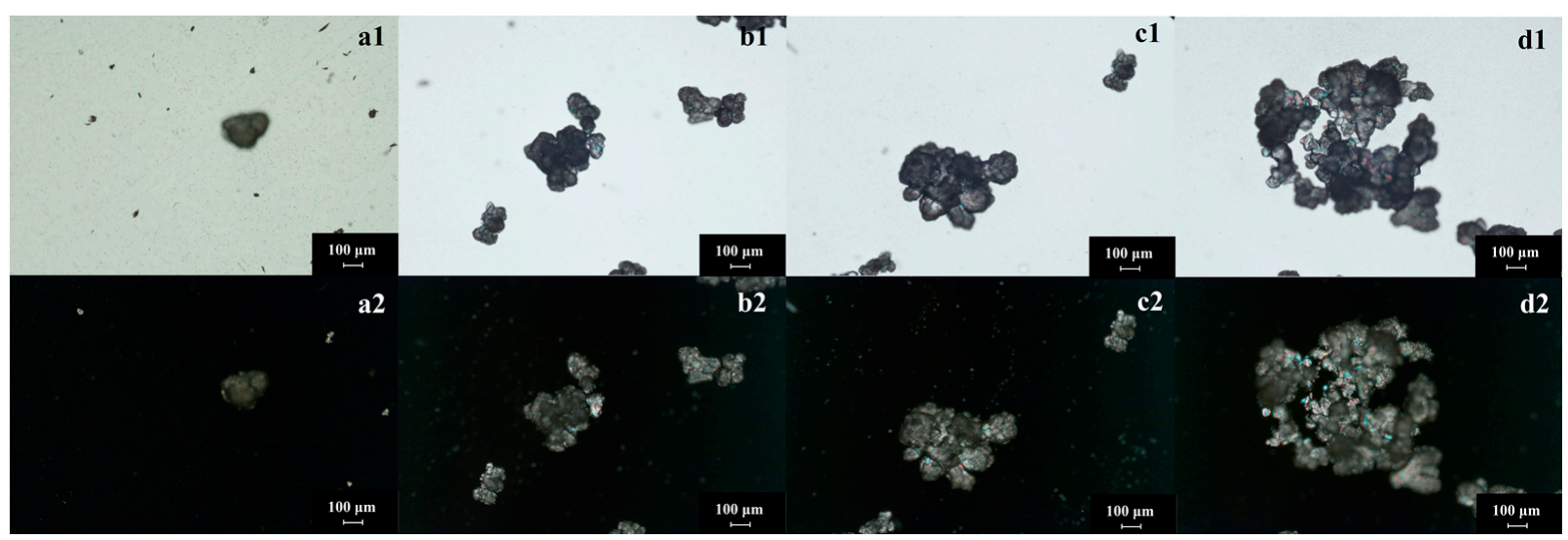

Figure 5. Image of the process of spontaneous nucleation to produce amorphous products. 
For GTA products, purity is an essential quality index. In the actual production process, epichlorohydrin would be recycled to reduce the cost. It was found in the previous experiment, when the epichlorohydrin used as the reactant was recovered, the purity, whiteness and particle size of the final product are difficult to control. Considering that epichlorohydrin is highly corrosive, general process analysis tools (e.g., process imaging system, ATR-FTIR or particle size tester) cannot be utilized in this reaction crystallization system. A polarizing microscope was selected to observe the crystallization process of GTA in this investigation. There are mainly three methods to prepare the production of GTA including (i) the amorphous products prepared by the spontaneous nucleation method, (ii) the crystalline products prepared by the spontaneous nucleation method, and (iii) the crystalline products prepared by the seed method.

i. Amorphous products prepared by the spontaneous nucleation method: Flow rate of the trimethylamine gas is the key factor affecting amorphous shape or crystallinity. When the flow rate is larger than $100 \mathrm{~mL} / \mathrm{min}$ in this investigation, the product would be amorphous. As shown in Figure 5, there is no polarization phenomenon of amorphous products (catch with XRD without diffraction peak). In this process, the solids are formed gradually and they grow slightly (Figure 5(a1-d1)). The main behavior be-tween solids is agglomeration. The product has no fixed morphology and no polarization phenomenon (Figure $5(\mathrm{a} 2-\mathrm{d} 2)$ ). On the other hand, the purity of amorphous products is low (less than $85 \%$ in fresh epichlorohydrin and less than $80 \%$ in the experiment of epichlorohydrin recycling). Amorphous solids tend to contain higher Gibbs free energy, which results in poor stability. Furthermore, amorphous solids are easy to preserve solvents (or reactant), which leading impurity content increased. GTA is a substance that absorbs moisture easily, especially in an amorphous state. Thus, amorphous GTA should be prevented during the reaction crystallization process.

ii. Crystalline products prepared by spontaneous nucleation method: The spontaneously nucleated GTA is a rod-like crystal with high crystallinity (as shown in Figure 6(a2-d2)). The spontaneous nucleation process is fast, with the constant addition of trimethylamine gas (Figure 6(a1,b1)). The rod-like crystals of GTA gradually multi-ply and aggregate (Figure 6(c1,d1)). The purity of the crystals produced by this method could be larger than $90 \%$, but the particle size is small. Solids agglomerate seriously during the storage procedure. Products prepared by the spontaneous nucleation method contains some uncertainty. The product may be crystalline or amorphous. When the reactant is recovered (epichlorohydrin), it is more difficult to obtain crystal products with high purity.

iii. Crystalline products prepared by the seed method: The seed method is widely used in the crystallization process. However, the application of seed in reaction crystallization-especially in gas- and liquid-phase reaction crystallization-is used relatively rarely. The investigation found that the seed method could produce high purity GTA solids no matter if the reactant of epichlorohydrin is recycled or not. The process involves first adding a certain amount of trimethylamine gas into epichlorohydrin (as mentioned in the experimental section). It must be emphasized that the seeds should be in crystal form (measured by XRD before using). After adding seed crystals, the seeds are aggregated pellets first (Figure $7(\mathrm{a} 1, \mathrm{~b} 1)$ ). At this time, the phenomenon of seed polarization is not obvious because the thickness of the solid is too large. Then, the seeds will spread out (Figure 7(a2,b2)). With the adding of trimethylamine gas, GTA grows and aggregates at the same time (Figure $7(\mathrm{c} 1, \mathrm{~d} 1)$ ). The crystal seeds regulate the number of crystal particles and the growth rate of crystal to a certain extent. The seed product has excellent crystallinity (Figure 7(c2,d2)) and pupurity (larger than $95 \%$ ). This method will be examined emphatically in the following section. 


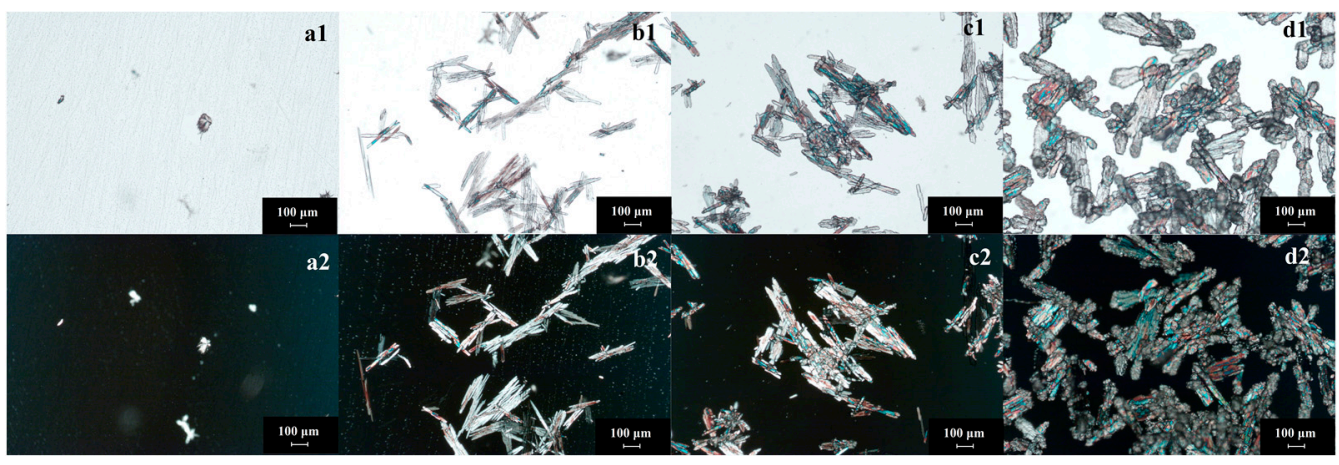

Figure 6. Image of the process of spontaneous nucleation to produce crystalline products.

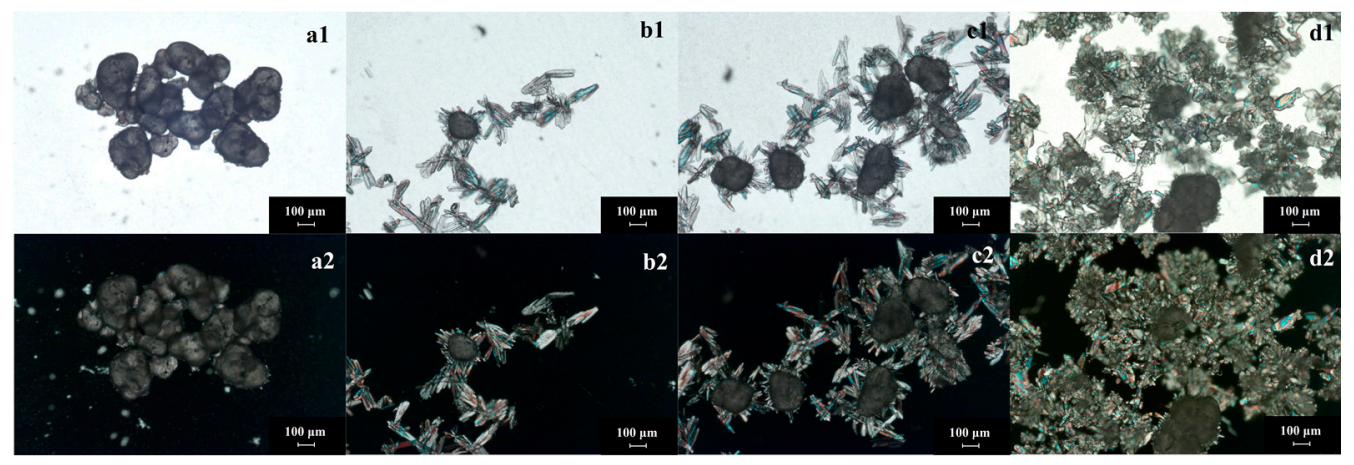

Figure 7. Image of the process of seed method to produce crystalline products.

\subsection{Understanding of the Crystallization Process}

The seed method is an effective way to control the purity of GTA solids. As mentioned, the seed crystals were obtained from spontaneous nucleation production. The way the crystals aggregate and grow is shown in Figure 8. Seed crystals were dispersed by the action of stirring. Then, with the increasing of trimethylamine content in the system, cystals began to grow on the basis of some rough crystallization sites on the seed surface (b1 in Figure 8). At this point, the seed plays the role of the growth core. The crystallization process of the gas-liquid reaction is rapid. The solid content in the system gradually increases. The generated GTA solids begin to grow and aggregate with seed as the center (d1 in Figure 7 and the "agglomeration and growth" step in Figure 8). In this process, seed plays a role in the growth and aggregation of the core. By this time, the growth rate of GTA would affect the purity. Excessive growth rates lead to the entrapment of reactants and impurities.

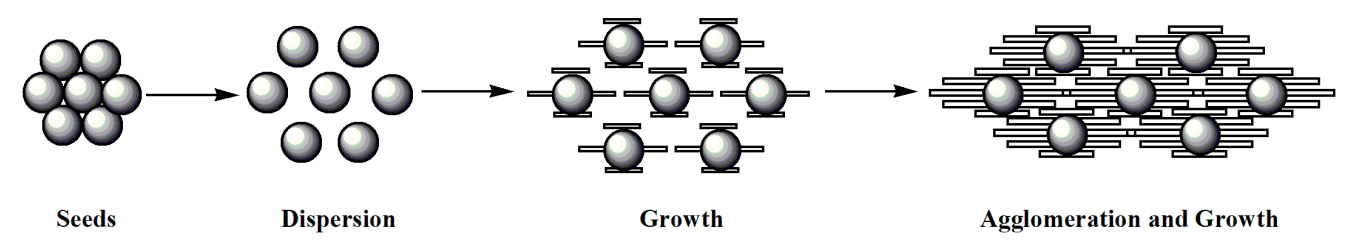

Figure 8. Agglomeration and growth of particles.

In summary, the reaction crystallization of GTA involves four steps. Firstly, approximately spherical seeds were added to the system. Then, aggregated seeds were dispersed. Thirdly, crystals on the seed surface began to grow. Finally, generated GTA solids began to grow and aggregate. 


\subsection{Planning of Experiments}

For the reaction crystallization, the reaction temperature and concentration of the reactant often play important roles; however, the reaction crystallization process of GTA has some particularity. The process has no real meaning for the solvent. Epichlorohydrin acts as a reactant and a dispersant. The other reactant is a gas state (trimethylamine). Therefore, factors including reaction temperature, flow rate of gas, stirring speed, seed amount and length of breeding time were investigated in this experiment. The details of experimental parameters are listed in Table 2.

Table 2. Experiments.

\begin{tabular}{cccccc}
\hline Experiment No. & $\begin{array}{c}\text { Reaction } \\
\text { Temperature }\left({ }^{\circ} \mathbf{C}\right)\end{array}$ & $\begin{array}{c}\text { Stirring Speed } \\
(\mathbf{r} / \mathbf{m i n})\end{array}$ & $\begin{array}{c}\text { Flow Rate } \\
(\mathbf{m L} / \mathbf{m i n})\end{array}$ & $\begin{array}{c}\text { Seed Amount } \\
\mathbf{( w t} \text { \%) }\end{array}$ & $\begin{array}{c}\text { Length of } \\
\text { Breeding Time (h) }\end{array}$ \\
\hline exps 1-5 & $10,15,20,25,30$ & 80 & 40 & 1.0 & 2 \\
exps 6-10 & 20 & $60,80,100,120,140$ & 40 & 1.0 & 2 \\
exps 11-16 & 20 & 100 & $30,40,50,60,70$ & 1.0 & 2 \\
exps 17-22 & 20 & 100 & 50 & $0.2,0.5,1.0,2.0,3.0$ & 2 \\
exps 23-27 & 20 & 100 & 50 & 1.0 & $1,2,3,4,5$ \\
exp 28 & 20 & 100 & 50 & 1.0 & 3 \\
exp 29 & 20 & 60 & 150 L/min & 1.0 & 4 \\
\hline
\end{tabular}

\subsection{Factors Affecting Purity}

Seed is the key factor affecting the purity of GTA. As shown in Table 3, when the seed method is used, the product tends to be of higher purity. The number of seeds can influence the quality of the final production (exps 17-22). With the increase in seed quantity, the purity of GTA increased first and then remained unchanged. When the seed amount is small, it is not enough to provide sufficient sites for growth and nucleation. There is a certain amount of spontaneous nucleation occurring, which leads to a decrease in purity. Solids formed by spontaneous nucleation tend to contain impurities and are less stable. On the other hand, the spontaneous nucleation products tend to be looser, which leads GTA to be more likely to degrade in contact with water and oxygen during storage. When the seed amount increases (larger than $1.0 \mathrm{wt} \%$ ), the total yield is constant; too many seeds will also lead to the reduction in the particle size of the final product. The larger specific surface of small particles will also lead to faster degradation. What is more, excess seed is also a cost loss.

Table 3. Purity of final products under different seed amount (calculated by Equation (1)).

\begin{tabular}{ccccccc}
\hline Seed Amount (wt \%) & $\mathbf{0}$ & $\mathbf{0 . 2}$ & $\mathbf{0 . 5}$ & $\mathbf{1 . 0}$ & $\mathbf{2 . 0}$ & $\mathbf{3 . 0}$ \\
\hline Purity of GTA (\%) & 89.4 & 95.7 & 96.4 & 97.3 & 97.0 & 97.1 \\
\hline
\end{tabular}

It is worth noting that when the reactant is fresh epichlorohydrin, a certain amount of trimethylamine gas is required before seed is added. This may be explained by the presence of GTA molecules in the pre-state system. Although GTA molecules did not separate out as a solid, this system is favorable for the existence and dispersion of seed. When the reactant is recycled epichlorohydrin, there is no need to include gas in advance because there is already a certain number of GTA molecules in the recovered epichlorohydrin system.

Another factor affecting purity is the flow rate of trimethylamine (exps 11-16). There is a negative correlation between product purity and flow rate. Excessive flow rate will make the crystal growth process speed up, which enhances the encapsulation of solvent and impurity. The byproducts [i.e., (2,3-dihydroxypropyl)trimethylammonium chloride, trimetlylamine oxide, biquaternary ammonium salt of GTA and GTA polymer] produced by the side reaction (e.g., hydrolysis, oxidation, polymerization and reverse reaction) would be wrapped in the GTA. All in all, the purity is affected by formation of new impurities, degrade of main product at a later stage, residues of reactants, etc. Larger flow rate 
also causes the crystal size to become tiny. Low flow rate will prolong the reaction time. Considering product purity and operation time, the flow rate of $50 \mathrm{~mL} / \mathrm{min}$ was selected (purity larger than 95\%).

\subsection{Factors Affecting Particle Size}

As mentioned above, both seed amount and flow rate have certain effects on particle size. Among all the factors, stirring speed has the greatest influence on particle size (exps 6-10). Due to the strong hygroscopicity of GTA, it is difficult to characterize the particle size by traditional methods. On the other hand, the particle size of GTA is almost larger than $500 \mu \mathrm{m}$, so the microscope is not suitable. As shown in Figure 9, with the increase in stirring rate, the particle size decreases (from approximate $2000 \mu \mathrm{m}$ to $500 \mu \mathrm{m}$ ). Because large particles have the advantages of storage and packaging, large particles are expected in the experiment. Rapid stirring speed increases the profile of collisions between gas-liquid reactants. Meanwhile, rapid stirring also increases the collisions between solid particles, which leads to increased nucleation rates and crystal fragmentation. When the stirring speed is less than $80 \mathrm{r} / \mathrm{min}$, with the increase in solid content in the later stage of the reaction, the solids will settle at the bottom of the crystallizer. In order to prevent solids from settling and affecting the crystallization reaction, the stirring speed should not be too low. Considering the degree of mixing and particle size, it is better to choose $100 \mathrm{r} / \mathrm{min}$ for laboratory level experiment.

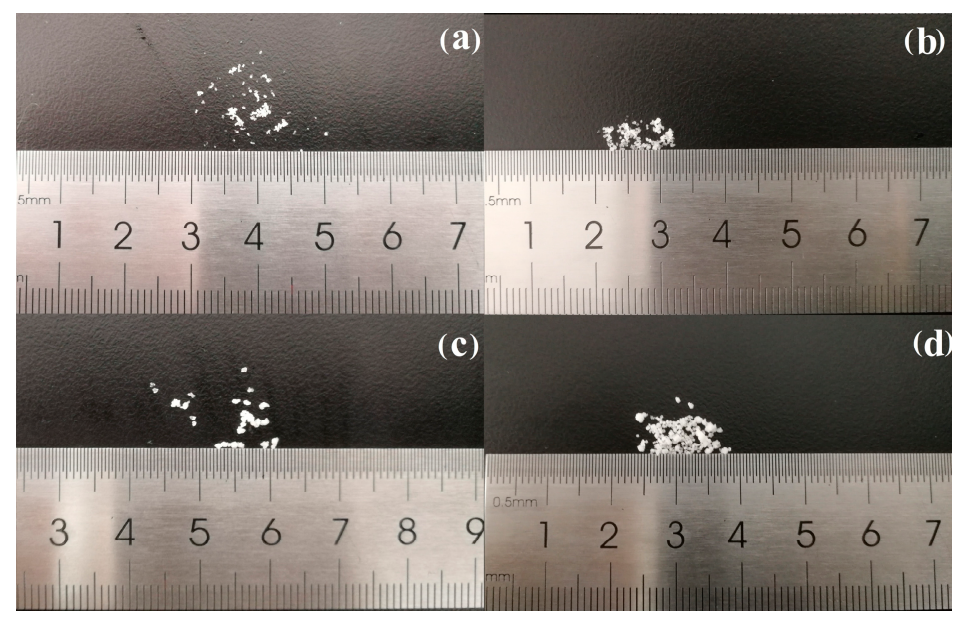

Figure 9. Particle images of final products of GTA under different stirring speeds ((a) $140 \mathrm{r} / \mathrm{min}$, (b) $120 \mathrm{r} / \mathrm{min}$, (c) $100 \mathrm{r} / \mathrm{min}$, (d) $80 \mathrm{r} / \mathrm{min}$ ).

\subsection{Factors Affecting Product Color and Yield}

The crystallization temperature has an important effect on the color of the product (exps 1-5). When the reaction temperature is higher than $25^{\circ} \mathrm{C}$, the product will be yellow. Therefore, the experimental temperature was selected below $30^{\circ} \mathrm{C}$. Higher temperature can accelerate the decomposition of GTA in the solution system and increase the degree of some side reactions. In general, solids suspended in solution would be significantly less stable than those in the nonliquid state. The presence of water and oxygen tends to accelerate the deterioration of GTA. Growth rate of the GTA crystal is slow at low temperatures (lower than $15^{\circ} \mathrm{C}$ ), which leading the crystallization period prolonged. The reaction rate is slow at low temperatures, and so is the growth rate of crystals. The length of breeding time of crystallization has an effect on the yield (exps 23-27). The length of breeding time is the guarantee of the degree of reaction. The residual free trimethylamine molecules in the system need to react with epichlorohydrin gradually. When the length of breeding time reaches $3 \mathrm{~h}$, the yield would be unchanged. 


\subsection{Process Identification and Scale-Up}

The optimized reaction crystallization condition in the $1 \mathrm{~L}$ scale reactor was as follows: reaction temperature, $20^{\circ} \mathrm{C}$; stirring speed, $100 \mathrm{r} / \mathrm{min}$; gas flow rate, $50 \mathrm{~mL} / \mathrm{min}$; number of seeds, $1 \mathrm{wt} \%$; length of breeding time, $3 \mathrm{~h}$ (exp 28). When the initial fresh epichlorohydrin is $600 \mathrm{~g}$, the crystal seed needs to be added after adding $1000 \mathrm{~mL}$ of trimethylamine gas into the reactor. The total time of adding gas in the second step is $3 \mathrm{~h}$. The purity of the final products is larger than $97 \%$ (the purity of the original process is less than $90 \%$ ). The particle size of the product will also increase significantly. The condition (exp 29) was successfully scaled up to $3000 \mathrm{~L}$ with satisfactory purity and particle size. According to the production test data, the gas flow rate is increased proportionately, stirring speed was $60 \mathrm{r} / \mathrm{min}$, and the length of breeding time was up to $4 \mathrm{~h}$. The purity of final products produced by scale-up experiments is larger than $97 \%$. The particle size is approximately $1500 \mu \mathrm{m}$. The yield and whiteness are basically the same as the laboratory level and they meet the product requirements.

\section{Conclusions}

The crystallization process of the GTA gas-liquid reaction was optimized by the seed method. The optimized technology can prepare GTA products with crystal form and purity greater than $97 \%$. The reaction process of GTA was analyzed. The crystallization process of GTA consists of four steps (i.e., addition of seed, dispersion of seed, growth of crystal, agglomeration and growth of crystal). Seed method and flow rate are the key factors affecting purity. Stirring speed has an obvious effect on particle size. The optimized reaction crystallization condition in the $1 \mathrm{~L}$ scale reactor: reaction temperature, $20{ }^{\circ} \mathrm{C}$; stirring speed, $100 \mathrm{r} / \mathrm{min}$; gas flow rate, $50 \mathrm{~mL} / \mathrm{min}$; number of seed, $1 \mathrm{wt} \%$; length of breeding time, $3 \mathrm{~h}$. The process can be successfully scaled up to $3000 \mathrm{~L}$. The purity and particle size of the product were improved obviously.

Author Contributions: Conceptualization, S.Y.; Methodology, S.Y.; Software, S.Y.; Validation, S.Y., F.X. and Y.C.; Formal Analysis, W.F.; Investigation, S.Y. and W.X.; Resources, S.Y.; Data Curation, H.C. and X.G.; Writing — Original Draft Preparation, S.Y.; Writing - Review \& Editing, S.Y.; Visualization, S.Y.; Supervision, F.X. and Y.C.; Project Administration, F.X. and Y.C.; Funding Acquisition, S.Y., F.X., W.F., Y.W. and S.D. All authors have read and agreed to the published version of the manuscript.

Funding: This research was funded by the Natural Science Foundation of Shandong Province (grant references: ZR2020QB177 and ZR2019QB020), the Science, Education and Industry Integration Technology Innovation Project (2020KJC-ZD09), the National Natural Science Foundation of China (NSFC) (grant references: 22008174 and 22008175), and the Taishan Scholars Program of Shandong, China (No. ts20130918).

Institutional Review Board Statement: Not applicable.

Informed Consent Statement: Not applicable.

Data Availability Statement: Not applicable.

Acknowledgments: The authors would like to extend their thanks to Pharmavision (Qingdao) Intelligent Technology Ltd. who provided assistance in the crystallization equipment.

Conflicts of Interest: The authors declare no conflict of interest.

\section{References}

1. McClure, J.D. Glycidyltrimethylammonium chloride and related compounds. J. Org. Chem. 1970, 35, 2059-2061. [CrossRef]

2. Bendoraitiene, J.; Kavaliauskaite, R.; Klimaviciute, R.; Zemaitaitis, A. Peculiarities of starch sationization with glycidyltrimethylammonium chloride. Starch Strke 2006, 58, 623-631. [CrossRef]

3. Cote, A.; Erdemir, D.; Girard, K.P.; Green, D.A.; Lovette, M.A.; Sirota, E.; Nere, N.K. Perspectives on the current state, challenges, and opportunities in pharmaceutical crystallization process development. Cryst. Growth Des. 2020, 20, 7568-7581. [CrossRef]

4. Wang, D.; Li, Z. Gas-liquid reactive crystallization kinetics of hydromagnesite in the MgCl2-CO2-NH3-H2O system: Its potential in $\mathrm{CO}_{2}$ sequestration. Ind. Eng. Chem. Res. 2012, 51, 16299-16310. [CrossRef] 
5. Kang, S.H.; Lee, S.G.; Jung, W.M.; Kim, M.C.; Kim, W.-S.; Choi, C.K.; Feigelson, R.S. Effect of Taylor vortices on calcium carbonate crystallization by gas-liquid reaction. J. Cryst. Growth 2003, 254, 196-205. [CrossRef]

6. Li, Y.; Zhang, Y.; Wang, X.Z. Secondary nucleation kinetics of AIBN crystallisation in methanol: Online imaging-based measurement and modelling. Crystals 2020, 10, 506. [CrossRef]

7. Liu, X.J.; Zhang, Y.; Wang, X.Z. Study on co-crystallization of LCZ696 using in situ ATR-FTIR and imaging. Crystals 2020, 10, 922. [CrossRef]

8. Li, K.; Wu, S.; Xu, S.; Du, S.; Zhao, K.; Lin, L.; Yang, P.; Yu, B.; Hou, B.; Gong, J. Oiling out and polymorphism control of pyraclostrobin in cooling crystallization. Ind. Eng. Chem. Res. 2016, 55, 11631-11637. [CrossRef]

9. Yu, S.; Xing, W.; Xue, F.; Cheng, Y.; Liu, Y.; Chen, H.; Hao, C.; Sun, Y. Measurement and correlation of solubility and thermodynamic properties of fluoxetine hydrochloride in 15 pure solvents and a methanol + water binary solvent system. J. Chem. Eng. Data 2020, 65, 4656-4668. [CrossRef]

10. Yu, S.; Xing, W.; Xue, F.; Cheng, Y.; Li, B. Solubility and thermodynamic properties of nimodipine in pure and binary solvents at a series of temperatures. J. Chem. Thermodyn. 2021, 152, 106259. [CrossRef]

11. Yu, S.; Xu, X.; Xing, W.; Xue, F.; Cheng, Y. Solubility, thermodynamic parameters, and dissolution properties of gliclazide in seventeen pure solvents at temperatures from 278.15 to 318.15 K. J. Mol. Liq. 2020, 312, 113425. [CrossRef]

12. Yu, S.; Yuan, J.; Cheng, Y.; Du, S.; Wang, Y.; Xue, F.; Xing, W. Solid-liquid phase equilibrium of clozapine in aqueous binary solvent mixtures. J. Mol. Liq. 2021, 329, 115371. [CrossRef]

13. Tacsi, K.; Pataki, H.; Domokos, A.; Nagy, B.; Csontos, I.; Markovits, I.; Farkas, F.; Nagy, Z.K.; Marosi, G. Direct processing of a flow reaction mixture using continuous mixed suspension mixed product removal crystallizer. Cryst. Growth Des. 2020, 20, 4433-4442. [CrossRef]

14. Takasuga, M.; Ooshima, H. Control of crystal aspect ratio and size by changing solvent composition in oiling out crystallization of an active pharmaceutical ingredient. Cryst. Growth Des. 2015, 15, 5834-5838. [CrossRef]

15. Li, X.; Yin, Q.; Zhang, M.; Hou, B.; Bao, Y.; Gong, J.; Hao, H.; Wang, Y.; Wang, J.; Wang, Z. Process design for antisolvent crystallization of erythromycin ethylsuccinate in oiling-out system. Ind. Eng. Chem. Res. 2016, 55, 7484-7492. [CrossRef]

16. Marone, P.A.; Thiyagarajan, P.; Wagner, A.M.; Tiede, D.M. Effect of detergent alkyl chain length on crystallization of a detergentsolubilized membrane protein: Correlation of protein-detergent particle size and particle-particle interaction with crystallization of the photosynthetic reaction center from Rhodobacter sphaeroides. J. Cryst. Growth 1999, 207, $214-225$.

17. Chen, C.W.; Lee, T. Round granules of dimethyl fumarate by three-in-one intensified process of reaction, crystallization, and spherical agglomeration in a common stirred rank. Org. Process Res. Dev. 2017, 21, 1326-1339. [CrossRef]

18. Testa, C.J.; Shvedova, K.; Hu, C.; Wu, W.; Born, S.C.; Takizawa, B.; Mascia, S. Heterogeneous crystallization as a process intensification technology in an integrated continuous manufacturing process for pharmaceuticals. Org. Process Res. Dev. 2021, 25, 225-238. [CrossRef]

19. Yu, S.; Zhang, Y.; Wang, X. Improved understanding of cefixime trihydrate reactive crystallization and process scale-up with the aid of PAT. Org. Process Res. Dev. 2019, 23, 177-188. [CrossRef] 